\title{
Genome wide analysis of the bovine mucin genes and their gastrointestinal transcription profile
}

\author{
Prisca R Hoorens ${ }^{1}$, Manuela Rinaldi ${ }^{1}$, Robert W Li ${ }^{2}$, Bruno Goddeeris ${ }^{3}$, Edwin Claerebout ${ }^{1}$, Jozef Vercruysse ${ }^{1}$, \\ Peter Geldhof ${ }^{*}$
}

\begin{abstract}
Background: Mucins are large glycoproteins implicated in protection of all mucosal surfaces. In humans and rodents, the mucin gene family has been well described and previous studies have investigated the distribution and function of mucins in the gastrointestinal (Gl) tract. In contrast, little data is available on the mucin gene family in polygastric species, such as cattle. The aim of the current study was to identify all members of the bovine mucin family by genome mining and subsequently investigate the transcription pattern of these mucins in the $\mathrm{Gl}$ tract.

Results: Nine bovine membrane-associated mucins (MUC1, MUC3A, MUC4, MUC12, MUC13, MUC15, MUC16, MUC20 and MUC21) and six secreted mucins (MUC2, MUC5AC, MUC5B, MUC6, MUC7 and MUC19) were identified in the bovine genome. No homologues could be identified for MUC3B, MUC8 and MUC17. In general, domain architecture of the membrane-associated mucins was found to be similar between humans and cattle, while the protein architecture of the gel-forming mucins appeared to be less conserved. Further analysis of the genomic organization indicated that the previously reported bovine submaxillary mucin (BSM) may be part of a larger gene encoding for MUC19. Analysis of the transcription profile showed that the secreted mucins were transcribed from the abomasum onwards, whereas the membrane associated mucins MUC1 and MUC20 were transcribed throughout the whole GI tract. In contrast to humans, MUC5B transcript was found in both the small and large intestine, but was absent in oesophageal tissue.
\end{abstract}

Conclusions: This study provides the first characterization of the mucin gene family in cattle and their transcriptional regulation in the Gl tract. The data presented in this paper will allow further studies of these proteins in the physiology of the $\mathrm{Gl}$ tract in ruminants and their interactions with pathogens.

\section{Background}

Mucins (MUC) are heavily O-glycosylated proteins that cover all mucosal surfaces. They play an important protective role as they form a physical, chemical and immunological barrier between the environment and the organism. Mucins can be largely divided into 2 structurally different families: the secreted and the membrane (cell surface)-associated mucins [1-4]. In humans, 7 members in the family of the secreted mucins have been identified, which can be further subdivided into gelforming mucins (MUC2, MUC5AC, MUC5B, MUC6, $M U C 19)$ and non-gel-forming mucins (MUC7, MUC8) [5-7]. The membrane-associated mucins, on the other

\footnotetext{
* Correspondence: peter.geldhof@ugent.be

'Department of Virology, Parasitology and Immunology, Faculty of Veterinary Medicine, Ghent University, Salisburylaan 133, 9820 Merelbeke, Belgium Full list of author information is available at the end of the article
}

hand, count 11 members (MUC1, MUC3A, MUC3B, MUC4, MUC12, MUC13, MUC15, MUC16, MUC17, MUC20 and MUC21) [8]. Structurally, all mucins have at least one mucin-like domain, named PTS-domain, formed by a variable number of tandem repeats (VNTR) rich in Pro, Thr and Ser residues [9]. These PTS domains carry the typical mucin O-glycosylations [10]. The secreted gel-forming mucins are further characterized by the presence of other typical domains [11], such as N-terminal TIL domains, $\mathrm{N}$ - and/or C-terminal Von Willebrand $\mathrm{D}$ and $\mathrm{C}$ domains (VWD/VWC), C8 domains, and a C-terminal cystine knot (CT) domain, all involved in the oligomerization through the formation of disulphide bridges between cysteine residues $[12,13]$. The capacity to form oligomers gives the secreted gel-forming mucins the ability to build up the dense, visco-elastic mucus gel that covers many
C Biomed Central 
epithelia [4,14-17]. Secreted non-gel-forming mucins on the other hand are not able to oligomerize, and their structural and functional properties are not well described $[18,19]$. The cell surface-associated mucins also do not oligomerize, but they are characterized by specific domains such as the C-terminal sea urchin sperm protein-enterokinase-agrin (SEA) domain, cleaved after translation, an epidermal growth factor (EGF) or EGF-like (EGF-L) domain involved in the three dimensional structure, and a transmembrane domain (TM). MUC4, a member of the cell surface-associated mucins, together with the typical domains described above, contains a variant of the VWD domain that lacks cysteines, as well as an adhesion associated domain (AMOP) with a possible role in cell adhesion, and a NIDO domain of unknown function. The membrane bound mucins are mostly present on the apical membrane of epithelial cells, where they have been suggested to play a role in cell signalling. Some of them can also be found in the mucus layer, together with the secreted ones, probably due to proteolytic cleavage $[20,21]$ or to the expression of secreted splicing variants [22,23].

Sequencing and annotation of mucin genes is known to be difficult due to the large size and repetitive structure of these molecules; moreover, among species, differences in the mucin gene family have been reported. Comparison between humans and mice, two mammals with a completed annotated genome [24], has shown that although the majority of mucins are commonly represented in both species, differences for few mucin genes are evident. In humans, for example, a MUC17 and two MUC3 genes (MUC3A and $M U C 3 B$ ) have been described, while in mice only one $M U C 3$ gene has been identified and it is still under debate if it is the homologue of human MUC3A or MUC17 [25-27]. Moreover, the $M U C 8$ gene has so far only been described in humans [28]. In ruminant species, such as cattle, only few mucin genes have been described to date (i.e. MUC1 [29-31], MUC15 [23,32] and bovine submaxillary mucin $(B S M)[33,34])$; therefore the first goal of the current study was to provide a comprehensive overview of annotated and non-annotated members of the bovine mucin family through database search and comparison with their potential human homologue. Since mucins play a fundamental role in the gastrointestinal (GI) tract defence mechanism, the distribution of the different mucins throughout the GI tract has been widely studied for monogastric mammalians $[2,35,36]$. However, in polygastric species, mucin distribution is still largely unclear. Therefore, the second goal of the current study was to investigate the transcriptional distribution of the identified bovine mucins throughout the GI tract of adult healthy cows.

\section{Methods}

\section{Identification and classification of mucin genes}

To identify mucin-encoding genes, the bovine genome assembly version Btau_4.0 was used, accessible through the NCBI database [37]. First, the database was termsearched for automatically annotated mucin genes. In a second step, mucin-encoding genes were also identified by BLASTn and BLASTp queries, using the nucleotide and amino acid sequences of 18 previously described human mucin molecules. These sequences included MUC1, MUC2, MUC3A, MUC3B, MUC4, MUC5AC, MUC5B, МUC6, MUC7, MUC8, MUC12, MUC13, MUC15, MUC16, MUC17, MUC19, MUC20 and $M U C 21$. The predicted bovine mucins were subsequently aligned with their putative human homologue to calculate amino acid sequence similarity. This was done using the MegAlign software (DNAStar).

In the situation where multiple partial bovine sequences were found with either the same annotation or showing similarity to the same human mucin sequence, the respective genomic and mRNA sequences were examined with SeqMan software (DNAStar) for possible overlaps. In the case of such overlap, the sequences were assembled to build up a contiguous genomic and transcript sequence and subsequently manually curated to identify open reading frames and protein sequences. The curations were based on comparisons with the human genomic and mRNA sequences, BLAST searches and bovine EST sequences, as well as sequencing of the overlap after PCR on samples collected from bovine GI tissues. When no overlap was found, a PCR approach was used on cDNA samples in order to investigate whether the predicted partial mucin sequences belonged to the same gene or transcript. Amplified fragments were extracted from agarose gel with the NucleoSpin ${ }^{\circledR}$ Extract kit (Macherey-Nagel), cloned into the pGEM $^{\circledR}$-T-Easy vector (Promega) and transformed to Escherichia coli DH5a cells (Invitrogen). Transformants were screened for inserts, according to the manufacturer's instructions and inserts were PCR-amplified with primers against the T7 and SP6 promoters located on the $\mathrm{pGEM}^{\circledR}$-T-Easy vector. PCR products were cleaned-up and sequence-analyzed at the Centre for Medical Genetics, Belgium [38]. All the new sequences identified were then submitted to the EMBL database as mRNA direct entries or as experimental TPA (Third Party Annotation) entries.

\section{Protein structure analysis}

The protein sequences of the human and bovine mucins were blasted against the SMART database [39] in the normal SMART mode, searching for Pfam domain and internal repeats. PTS regions were identified either by 
SMART as regions of low complexity [19] or by hand by calculating the percentage of $\mathrm{P}+\mathrm{T}(>40 \%)$ and $\mathrm{S}(>5 \%)$ residues in the repeats. Protein sequences were analyzed for signal sequences using the SignalP 3.0 server [40].

\section{Sample collection}

Tissue samples were obtained at the slaughter house from four healthy cows, i.e. two Holstein and two mixed breed animals (Belgian Blue White - Holstein). The animals selected were four years old with an average weight of $455 \mathrm{~kg}$. Oesophagus, rumen, reticulum, omasum, abomasum (fundic and pyloric region), duodenum, jejunum, ileum, caecum, colon and rectum from the GI tract were included. Tissues were snap frozen on liquid nitrogen and stored at $-80^{\circ} \mathrm{C}$ until RNA was extracted.

\section{RNA and genomic DNA extraction}

Total RNA was extracted from tissue samples using the RNeasy Mini kit (Qiagen). To remove contaminating genomic DNA (gDNA), on-column DNase digestion was performed using the RNase-free DNase set (Qiagen) according to the manufacturer's instructions. RNA quality was verified using an Experion ${ }^{\mathrm{TM}}$ Automated Electophoresis System (Bio-Rad), and concentrations were determined using a NanoDrop ND-1000 spectrophotometer (NanoDrop Technologies). For all samples, the RNA quality indicator (RQI) calculated by the Experion ${ }^{\mathrm{TM}}$ software (Bio-Rad) was $>8.0$, indicating high RNA integrity.
Genomic DNA contamination was investigated with the SuperScript One-Step RT PCR kit (Invitrogen) using intron-spanning primers for glyceraldehyde-3-phosphate dehydrogenase (GAPDH) (Table 1). Genomic DNA was extracted from one tissue sample using the DNeasy Blood and Tissue Kit (Qiagen) as recommended by the manufacturer and it was used as a positive control for primer specificity in subsequent PCR reactions.

\section{cDNA synthesis and PCR}

One $\mu \mathrm{g}$ of total RNA was converted to cDNA using the iScript cDNA synthesis kit (Bio-Rad), following the manufacturer's instructions. Primers used to perform the mucin PCRs are listed in Table 1 and were designed with an annealing temperature of $60^{\circ} \mathrm{C}$ using the Primer3 software [41]. PCR amplifications were carried out using the GoTaq ${ }^{\circledR}$ Flexi DNA Polymerase kit (Promega). A total reaction volume of $25 \mu \mathrm{l}$ was used, containing 1 $\mu \mathrm{l}$ of single-stranded cDNA (5 $\mathrm{ng}$ of the input total RNA equivalent); $0.4 \mu \mathrm{M}$ forward primer; $0.4 \mu \mathrm{M}$ reverse primer; $0.65 \mathrm{U} \mathrm{GoTaq}^{\circledR}$ DNA Polymerase; 0.2 $\mathrm{mM}$ each dNTP (PCR Nucleotide Mix); $1.5 \mathrm{mM} \mathrm{MgCl}_{2}$, 1x Green GoTaq ${ }^{\circledR}$ Flexi Buffer. The PCR reactions were cycled as followed: an initial denaturing step of $4 \mathrm{~min}$ at $95^{\circ} \mathrm{C}$, followed by 35 cycles of $30 \mathrm{~s}$ at $95^{\circ} \mathrm{C}, 30 \mathrm{~s}$ at $60^{\circ} \mathrm{C}$, $30 \mathrm{~s}$ at $72^{\circ} \mathrm{C}$ and a final extension of $10 \mathrm{~min}$ at $72^{\circ} \mathrm{C}$. PCR products were analyzed by electrophoresis in $1.5 \%$ agarose (Promega, Madison) gel at a constant voltage of

Table 1 Primer sequences and amplicon length

\begin{tabular}{|c|c|c|c|}
\hline Gene & Forward Sequence & Reverse Sequence & $A L^{a}(b p)$ \\
\hline $\mathrm{GAPDH}^{\mathrm{b}}$ & GGGTCATCATCTCTGCACCT & GGTCATAAGTCCCTCCACGA & 176 \\
\hline GAPDH & ACCCAGAAGACTGTGGATGG & CAACAGACACGTTGGGAGTG & 178 \\
\hline ACTB & GACATCCGCAAGGACCTCTA & ACATCTGCTGGAAGGTGGAC & 205 \\
\hline MUC1 & CACTGCTGCCAGCCATATTA & TCAAACCCCAAATGCTTCTC & 212 \\
\hline MUC2 & CATGTGGAACCAGGAGGACT & ATGTTCCCAACCTCGACAGG & 153 \\
\hline MUC3A & CCGTCACAAGTACATCTAACACAG & ACATATTCGAGGCGTTAGCA & 150 \\
\hline MUC4 & GGAGAGTGCAGAGTCCTTGG & AGCAGCAAAGCCAATGAAGT & 323 \\
\hline MUC5AC & CAGACCCTCCACCTTCTTCA & GGTCCTCGAAGCTGTTCTTG & 263 \\
\hline MUC5B & TCTACCTGACCGTGGAGACC & GTTGATGATGCTGCACTGCT & 299 \\
\hline MUC6-1 & CAGCAAGGACAAAATCGTGA & CTCTGGTCTGGCCTCTGAAC & 218 \\
\hline MUC6-2 & CACGGCCTCCTGTCTTCTAC & AGAAGATGGACTGGCTCTCA & 190 \\
\hline MUC7 & TCCTGCTCCTAAGGCTACCA & GTGGAGGGGAGTGGTACTGA & 155 \\
\hline MUC12 & TACAGGGGCAACAACTTTCC & CGTCTCATCGTAGCACAGGA & 234 \\
\hline MUC13 & ACCTGGGACAGAGACACCAC & GATTGTCGGGGTAGGAGACA & 262 \\
\hline MUC15 & CTGCCTTGGAACTCATCCAT & CACAGACGTGGTGTTTGGTC & 183 \\
\hline MUC16 & TTCAGAAACAGCAGCATTGG & GTGTAGTGGTCCAGCCGAGT & 200 \\
\hline MUC19 & TTCTGGAGGGGCTGAATATG & СTCTCGTCCACCAAAAGAGC & 204 \\
\hline MUC20 & GGACATCACTGCTCTGACGA & GGGCTATTGTCCAGGTCTCA & 239 \\
\hline MUC21 & CAACCACAGGAGGCTCTGAT & CAAAGAATCCCACGACCACT & 173 \\
\hline BSM & GGAGACATATGGACTGCCAAT & CACAGTATGCAATTTCACAGCA & 194 \\
\hline
\end{tabular}

${ }^{a} \mathrm{AL}$ : CDNA amplicon length.

${ }^{b}$ GAPDH intron-spanning primers, used to investigate gDNA contamination. 
$110 \mathrm{~V}$ with $1 \mathrm{X}$ Tris-acetate EDTA (TAE) buffer. DNA was visualised by ethidium bromide and analyzed using Image Quant ${ }^{\mathrm{TM}}$ 350. Image background was subtracted using the rolling ball method and band volumes were normalized against the known volume of the ladder 500 bp band (100 bp ladder, Promega) in each gel. Values obtained were arbitrarily grouped and bands classified as absent (green), present weak (yellow) and present strong (red). To control the quality of the synthesized cDNA in all the samples and to validate the classification based on band intensity, PCR on two housekeeping genes, GAPDH and $\beta$-actin $(A C T B)$, was also performed.

\section{Results}

Cell surface associated mucins

Searching the latest bovine genome assembly resulted in the identification of 10 sequences encoding 9 different cell surface associated mucins (Table 2). The percentage similarity, at amino acid level, between the predicted bovine mucins (bMUC) and their human homologues is reported in Table 2. A single bovine gDNA sequence encoding a predicted mRNA sequence was found for MUC1, MUC3A, MUC4, MUC12, MUC13, MUC15, $M U C 20$ and MUC21 (Table 2). Two MUC16-like sequences were identified and no bovine homologous sequences were found for human (h)MUC17 and $\mathrm{h} M U C 3 B$. Although all these bovine mucins showed low $(<70 \%)$ similarity with their human homologues, the overall protein architecture was found to be conserved (Figure 1), with the exception of MUC3A, MUC12 and MUC13.

The predicted bMUC3A was found to contain a signal peptide (SP) and a PTS repeat region. However, the Cterminal EGF, SEA and TM domains, present in the hMUC3A protein sequence, were not found in the predicted sequence (Figure 1). Analysis of bMUC12 on the

Table 2 Bovine and human mucin genes and protein similarity

\begin{tabular}{|c|c|c|c|c|c|c|c|c|}
\hline \multirow[b]{2}{*}{ Gene } & \multicolumn{3}{|c|}{ Bos taurus } & \multicolumn{4}{|c|}{ Homo sapiens } & \multirow[b]{2}{*}{$\%$} \\
\hline & CDS & gDNA & mRNA & Gene & CDS & gDNA & mRNA & \\
\hline \multicolumn{9}{|c|}{ Membrane-associated mucins } \\
\hline MUC1 & $\mathrm{FL}$ & NC_007301 & NM_174115 & MUC1 & $\mathrm{FL}$ & NC_000001 & NM_002456 & 62.4 \\
\hline MUC3A & $3^{\prime} p$ & NC_007326 & XM_001255602 & MUC3A & $\mathrm{FL}$ & NC_000007 & XM_001725354 & 12.4 \\
\hline- & & & & MUC3B & $\mathrm{FL}$ & AC_000050 & XM_001125753 & ND \\
\hline MUC4 & $5^{\prime} p$ & NC_007299 & XM_001788490 & MUC4 & $\mathrm{FL}$ & NC_000003 & XM_018406 & 66.6 \\
\hline MUC12 & $\mathrm{FL}$ & NC_007326 & BC142358 & MUC12 & $\mathrm{FL}$ & NC_000007 & NM_001164462 & 68.2 \\
\hline MUC13 & $\mathrm{FL}$ & NC_007299 & XM_865756 & MUC13 & $\mathrm{FL}$ & NC_000003 & NM_033049 & 19.6 \\
\hline MUC15 & $\mathrm{FL}$ & NC_007313 & NM_176631 & MUC15 & $\mathrm{FL}$ & NC_000011 & NM_001135091 & 65.9 \\
\hline MUC16 & $\mathrm{FL}$ & BN001315 & BN001315* & MUC16 & $\mathrm{FL}$ & NC_000019 & NM_024690 & 57.6 \\
\hline - & & & & MUC17 & $\mathrm{FL}$ & NC_000007 & NM_001040105 & ND \\
\hline MUC20 & $\mathrm{FL}$ & NC_007299 & XM_580797 & MUC20 & $\mathrm{FL}$ & NC_000003 & NM_152673 & 35.5 \\
\hline MUC21 & $\mathrm{FL}$ & NC_007324 & XM_001255727 & MUC21 & $\mathrm{FL}$ & NC_000006 & NM_001010909 & 38.4 \\
\hline \multicolumn{9}{|c|}{ Secreted mucins } \\
\hline \multirow[t]{2}{*}{ MUC2 } & $\mathrm{FL}$ & NW_001494549 & BN001490* & MUC2 & $\mathrm{FL}$ & NC_000011 & NM_002457 & 44.8 \\
\hline & & NW_001027928 & & & & & & \\
\hline MUC5AC & $\mathrm{FL}$ & BN001491* & BN001491* & MUC5AC & $\mathrm{FL}$ & NC_000011 & LT200503** & 33.2 \\
\hline MUC5B & $\mathrm{FL}$ & BN001492* & BN001492* & MUC5B & $\mathrm{FL}$ & NC_000011 & NM_002458 & 23.6 \\
\hline MUC6-1 & $3^{\prime} p$ & NW_001027928 & XM_867810 & MUC6 & $\mathrm{FL}$ & NC_000011 & NM_005961 & 61.4 \\
\hline MUC6-2 & $3 \prime p$ & NW_001494551 & XM_870967 & MUC6 & $\mathrm{FL}$ & NC_000011 & NM_005961 & 34.0 \\
\hline MUC7 & $3^{\prime} \& 5^{\prime} p$ & NW_001505809 & XM_001255642 & MUC7 & $\mathrm{FL}$ & NC_000004 & NM_152291 & 38.4 \\
\hline- & & & & MUC8 & $5^{\prime} p$ & - & U14383 & ND \\
\hline MUC19 & $5 \prime p$ & BN001489* & BN001489* & MUC19 & $3^{\prime} p$ & NC_000012 & XM_002343162 & 64.8 \\
\hline$B S M$ & $5^{\prime} p$ & NC_007303 & XM_615336 & MUC19 & $5^{\prime} p$ & NC_000012 & XM_002343163 & 50.7 \\
\hline
\end{tabular}

CDS: coding sequence.

gDNA: genomic DNA accession number in GenBank.

mRNA: messenger RNA accession number in GenBank.

$\%$ : percentage amino acid similarity between human and bovine mucin homologue.

FL: full length.

3'p: 3'partial, 5'p: 5'partial.

ND: not determined.

*EMBL accession number assigned to the newly generated sequences.

**mRNA sequence retrieved from the Mucin Biology Group mucin database [71]. 
other hand, did show the presence of the C-terminal SEA, EGF-L and TM domains, as seen in hMUC12, but there was no N-terminal SP or PTS region (Figure 1). At genomic level, bMUC3A is located immediately upstream of bMUC12 on chromosome 25 and has the same orientation. However, there is gap in the genomic sequence of about $5 \mathrm{~kb}$ between both genes, explaining why the 3'end of bMUC3A and the 5'end of bMUC12 are still missing. For bMUC13, a SP and PTS repeat have been identified, but no other domains present in hMUC13 have been found. Although the bMUC13 nucleotide sequence (GenBank: XM_865756) was discontinued from the database, transcription of bMUC13 was evident in GI tissue samples. However, since bMUC13 has not been mapped on the current genome build Btau_4.0, no further analysis to identify a putative 3'end was performed.

Two partial bMUC16 mRNA (GenBank: XR_042722, GenBank: XM_001254224), together with their respective gDNA sequences (GenBank: NC_007305.4 and GenBank: NC_007305.2), were found in the database. Alignment of these sequences showed an overlapping region in both the gDNA and mRNA sequences, which was subsequently confirmed by PCR and sequencing. The new full-length gDNA and mRNA sequence was submitted to EMBL under accession number EMBL: BN001315 (Table 2). Comparison of the domain architecture of bMUC16 with the human homologue showed that the protein structure was largely conserved (Figure 1), with the exception of a signal peptide only found in the bovine sequence.

\section{Secreted mucins}

Twelve bovine gDNA and predicted mRNA sequences were found in the sequence dataset, predicted to encode 7 secreted mucins (Table 2). Gel-forming MUC2, MUC5AC, $M U C 5 B, M U C 6, M U C 19, B S M$ as well as the non gel-forming $M U C 7$ were identified. No putative bovine homologue was found for human MUC8 (Table 2).

Two partial bovine $M U C 2$-like mRNA sequences (GenBank: XM_598117 and GenBank: XM_001256289), together with their corresponding gDNA (GenBank: NW_001027928 and GenBank: NW_001494549), were found in the database. Alignment with the human $M U C 2$

\section{MEMBRANE-ASSOCIATED MUCINS}

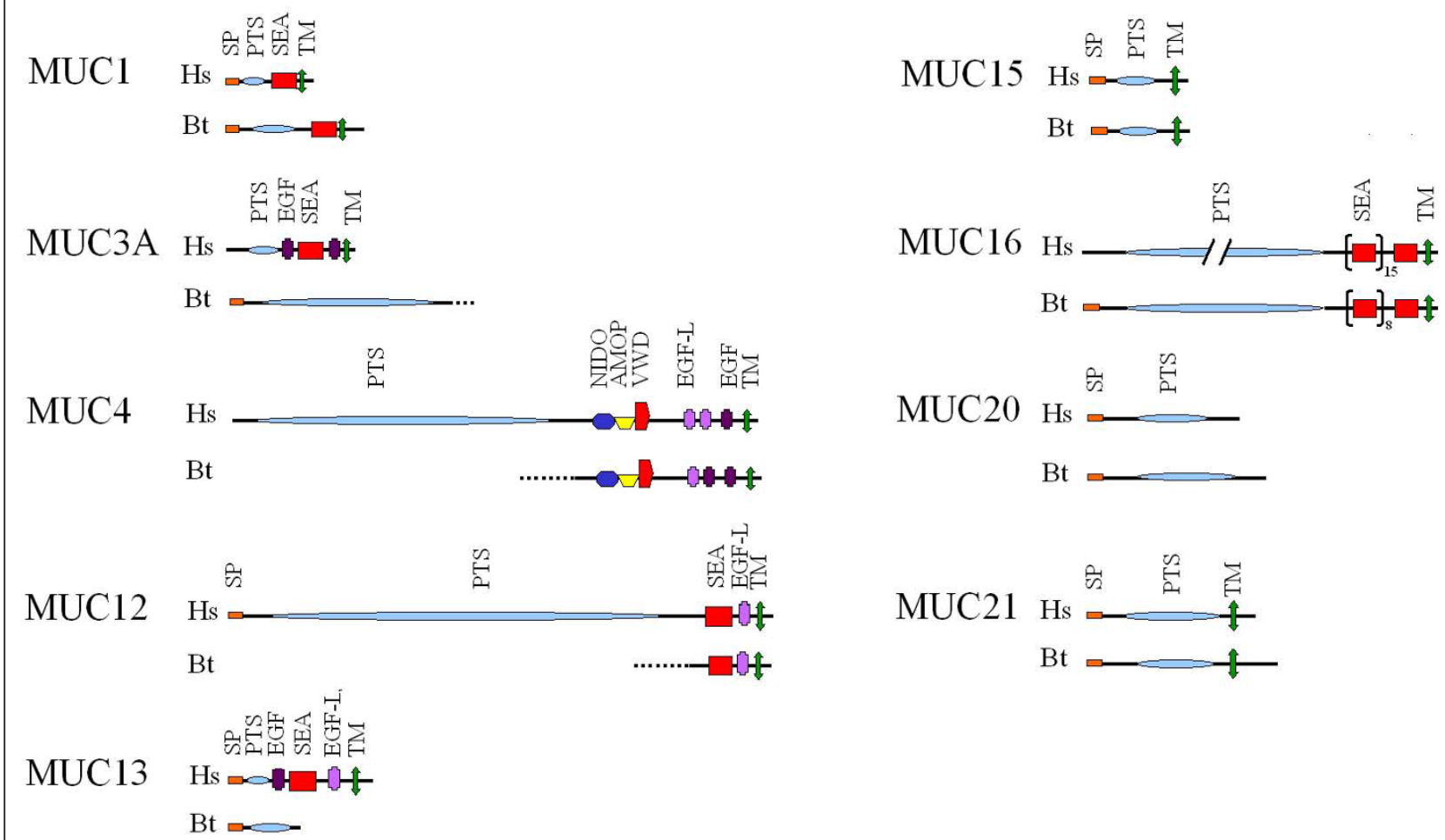

Figure 1 Protein architecture of the bovine cell surface associated mucins. Protein architecture of the human ( $\mathrm{Hs})$ and bovine (Bt) cell surface associated mucins MUC1, MUC3A, MUC4, MUC12, MUC13, MUC15, MUC16, MUC20 and MUC21. MUC16 is presented as a shortened sequence, indicated by //. Domains detected by SMART [39] are SP: signal peptide; PTS: Pro, Thr and Ser rich region; SEA: sea urchin sperm protein-enterokinase-agrin, TM: transmembrane domain; EGF: epidermal growth factor domain; EGF-L: EGF-like domain; NIDO: extracellular domain of unknown function; AMOP: domain involved in cell adhesion; WWD: Von Willebrand Factor D. 
homologue showed that one $\mathrm{b} M U C 2$-like mRNA sequence (GenBank: XM_598117) aligned to the 5'end of hMUC2 and the other one (GenBank: XM_001256289) to the 3'end, with no overlapping region. In an attempt to identify and sequence the missing central part of bovine $M U C 2$ mRNA, a PCR was performed on cDNA samples of the colon using a primer set spanning the missing region. The amplicon obtained in the PCR was subsequently cloned and sequenced. The two predicted sequences and the newly experimentally identified sequence were aligned and used to compose a new full-length bMUC2 mRNA sequence that was submitted to EMBL under the accession number BN001490 (Table 2). At genomic level, the same approach was taken but without success. Further blast search of the mRNA sequence against the bovine genome did not result in the identification of this region; therefore the gDNA sequence encoding the identified central part of the MUC2 mRNA still remains unknown.

Two predicted bMUC5-like sequences (GenBank: XM_604045 and GenBank: XR_042814) were found in the database. Sequence analysis indicated that the mRNA sequence XM_604045 showed the highest similarity to $\mathrm{h} M U C 5 A C$ and was therefore annotated as bMUC5AC in the EMBL database (EMBL:BN001491) (Table 2). The predicted mRNA sequence with GenBank accession number XR_042814 showed the highest similarity to $\mathrm{h} M U C 5 B$. At genomic level, bMUC5AC and $\mathrm{b} M U C 5 B$ sequences were found to overlap, indicating that the $\mathrm{b} M U C 5 A C$ and $\mathrm{b} M U C 5 B$ genes are located in $\mathrm{a}$ cluster on chromosome 29. According to the database, exons 1 to 7 of the predicted $\mathrm{b} M U C 5 B$ mRNA sequence were identical to exons 4 to 10 of the predicted $\mathrm{b} M U$ $C 5 A C$ mRNA. To investigate whether $\mathrm{b} M U C 5 A C$ and
bMUC5B are indeed alternatively spliced, or whether this has been wrongly annotated, the 5' end of the $\mathrm{b} M U C 5 B$ genomic sequence was analyzed for putative exons using the GENSCAN server [42]. Eight putative exons were identified encoding $M U C 5 B$ like sequences. Transcription of this newly identified bMUC5B 5'end was experimentally shown by PCR amplification on cDNA. The corrected bMUC5B mRNA sequence has now been submitted under accession number BN001492 (Table 2).

At protein level, the predicted bMUC5AC showed an overall similarity of $33.2 \%$ with the human homologue, although the similarity increases if comparing the $\mathrm{N}$-terminal region (amino acid AA 1-1350) before the PTS repeats $(70.3 \%)$ and the $\mathrm{C}$-terminal region (AA 2800-3554) after the PTS repeats (58.3\%) (Figure 2). Similarly, bMUC5B showed a low overall similarity $(23.6 \%)$ with the human homologue, but $75.2 \%$ and $53.2 \%$ similarity at the N-terminal region (AA 1-1040), and the C-terminal region (AA 5800-6725) respectively (Figure 2). Some domains of the hMUC5AC and hMUC5B protein are not found in bMUC5AC and bMUC5B (Figure 2). hMUC5AC has an N-terminal SP, VWD, C8, TIL and VWC domain (AA 1-443), of which TIL is not present in bMUC5AC (AA 1-459). These domains are followed by a second C8, TIL and VWC domain in hMUC5AC (AA 624-827), while only the second $\mathrm{C} 8$ domain in this region is found in bMUC5AC. A second VWD and third C8 domain before the PTS region are present both in hMUC5AC (AA 892-1162) and bMUC5AC (AA 893-1161). At the C-terminus of hMUC5AC, a VWC, VWD, C8, a second and third VWC and CT domain have been found, but in

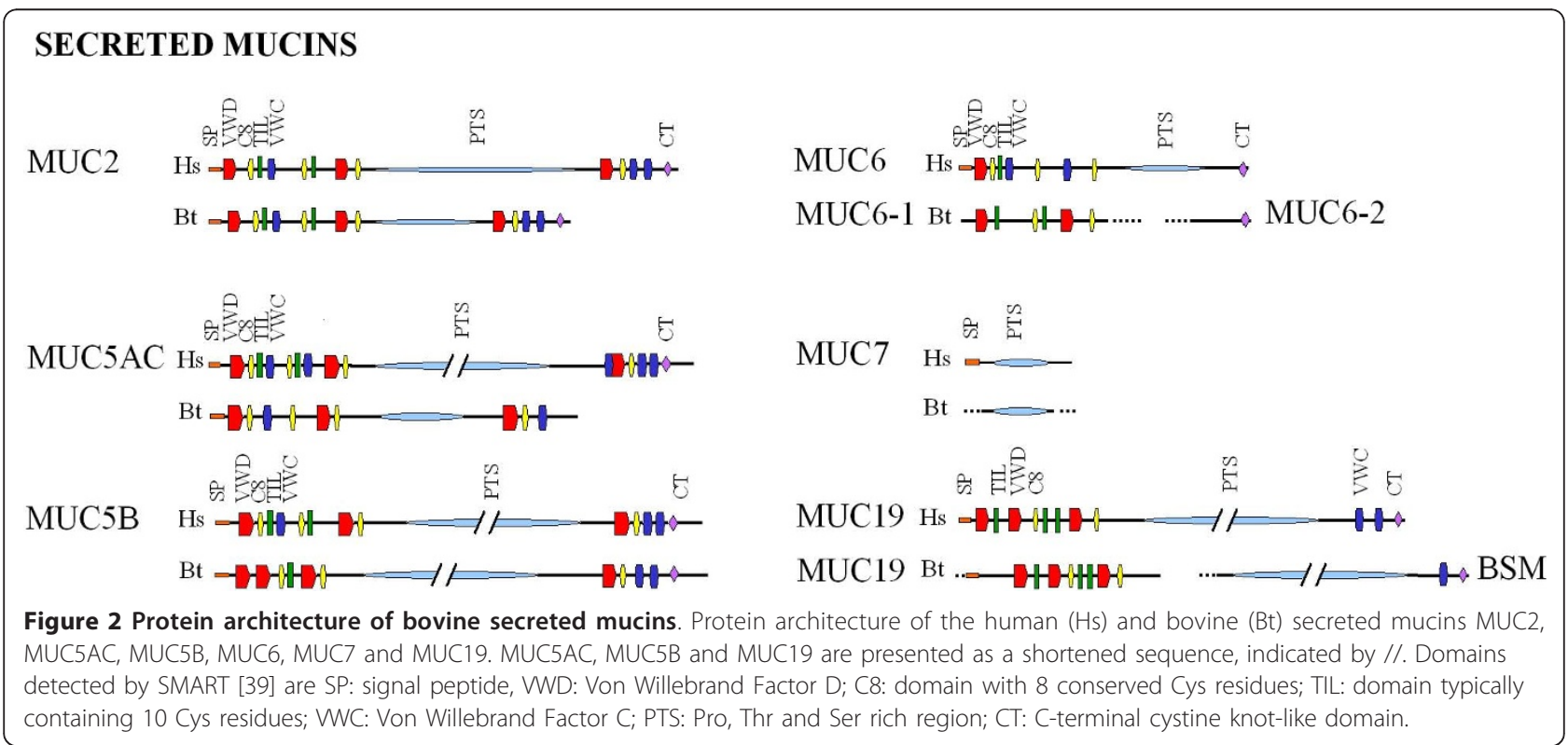


bMUC5AC, only the VWD, C8 and a VWC corresponding to the third VWC in hMUC5AC have been identified. Likewise, the organization of the domains is different for hMUC5B and bMUC5B. While hMUC5B contains the typical N-terminal SP, VWD, C8, TIL, VWC domain organization (AA 1-458), only the SP and VWD (AA 1- 216) have been conserved in bMUC5B, while the C8, TIL and VWC from hMUC5B have been replaced with a second VWD in bMUC5B (AA 234401). The domain organization of the remaining protein sequence is similar between hMUC5B and bMUC5B.

Two partial mRNA sequences were found in the database for MUC6. One bovine sequence (bMUC6-1; GenBank: NW_001027928) showed to align to the 5' end of hMUC6 and one (bMUC6-2; GenBank: NW_001494551) to the 3'end. For both $M U C 6$-like sequences corresponding gDNA sequences were also identified in the database. The alignment of all the bovine sequences found did not show any overlap at mRNA or gDNA level. Therefore, an attempt to identify the missing central region was done using a PCR approach, but without success. Although the similarity between hMUC6 and the N-terminal part of bovine MUC6-1 (AA 1-1170) is 70.8\%, the domain organization was found to be different (Figure 2). In humans, SP, VWC, C8, TIL and VWC domains (AA 1-427) are present in the $\mathrm{N}$-terminal region followed by $\mathrm{C} 8$, VWC and $\mathrm{C} 8$ domains. Instead, in bovine MUC6-1 only a VWD and a TIL domain are present in the N-terminal region followed by C8, TIL and VWD domains. In the central part of hMUC6 there is a PTS repeat region, which is still not identified in cattle (Figure 2).

Three partial sequences with similarity to human MUC19 were identified in GenBank. Two sequences (GenBank: XM_001788043 - similar to submaxillary apomucin, GenBank: XM_603306 - similar to MUC19) were found to align to the 5 ' end of $\mathrm{h} M U C 19$. Alignment of these two sequences showed an overlap at both the mRNA and gDNA level; hence a longer 5' partial bMUC19 (gDNA and mRNA) was constructed and submitted to the database under accession number BN001489. The third sequence identified in the database was the one encoding for bovine submaxillary mucin (BSM, GenBank: XM_615336). BSM is one of the few bovine mucin previously described $[33,34,43]$ and it was found to have a high similarity $(74 \%$ on amino acid level) with the C-terminal part of hMUC19 at protein level. The predicted $b M U C 19$ and BSM genes are both on chromosome 5 in cattle, separated by about $9 \mathrm{~kb}$ of genomic sequence, suggesting that BSM together with the predicted $b M U C 19$ might belong together and form one unique $M U C 19$ gene. To confirm this hypothesis the genomic sequence between BSM and bMUC19 was analyzed using the GENSCAN server [42]. Only short exons (21-167 bp, at suboptimal cut-off level 0.01) could be predicted in this region and none of them encoded for mucin domains or PTS-rich peptides. Also in humans the central PTS region of MUC19 is unknown, but a putative full-length $h M U C 19$ has been proposed by Chen et al. (2004) [44] (Figure 2). On the other hand in mice and in pigs full-length genes homologous to hMUC19 have been previously identified, named Muc19 and porcine submaxillary mucin (PSM), respectively. At protein level, alignment of both BMUC19 and BSM with human, mouse and pig MUC19 suggests that all the expected domains, typical for MUC19, are represented in bMUC19 and BSM. Assuming that the complete bovine MUC19 has a similar length compared to PSM and mouse Muc19, it would mean that the unknown region between BSM and bMUC19 is about 500 AA long. For this reason PCR was performed on jejunal samples in an attempt to identify this putative linkage region. However, no amplification was obtained, likely due to the presence of a repetitive sequence encoding the PTS-rich regions.

\section{Membrane associated and secreted mucins are differentially transcribed in the gastrointestinal tract}

To understand the transcription profile for all the known bovine mucins in the gastro-intestinal tract, $\mathrm{PCR}$ was performed on cDNA of samples collected from several tissues. The results obtained showed a characteristic transcription profile for many of the mucins analyzed in both the membrane associated and the gel-forming groups (Figure 3). Among the membrane associated mucins, MUC1 and MUC20 were majorly transcribed, with occurrence of bands in all the tissues examined. $M U C 15, M U C 16$, and $M U C 21$ were found to be transcribed in the first part of the GI tract with the strongest presence in the oesophagus of all the animals analyzed, while $M U C 3 A$ and $M U C 13$ were transcribed in the small and large intestine only. In contrast, MUC12 was hardly present in any of the tissues analyzed with only weak bands in few samples collected from the large intestine. MUC4 transcription was only detected in two samples collected in the oesophagus and one in the fundic region of the abomasum, caecum, colon and rectum.

In regard to the secreted gel-forming mucins, MUC5AC and MUC6 (both MUC6-1 and MUC6-2) were found to be consistently transcribed in the abomasum, both fundic and pyloric regions, and in the first part of the intestinal tract (duodenum and jejunum). $M U C 2$ and $M U C 5 B$ were only transcribed in the intestinal section of the GI tract with bands present in the majority of the tissues collected from this area. MUC19 and $B S M$ showed a similar transcription pattern, with weak to strong bands in 3 animals in the jejunum and weak transcription in one sample in the rectum. 


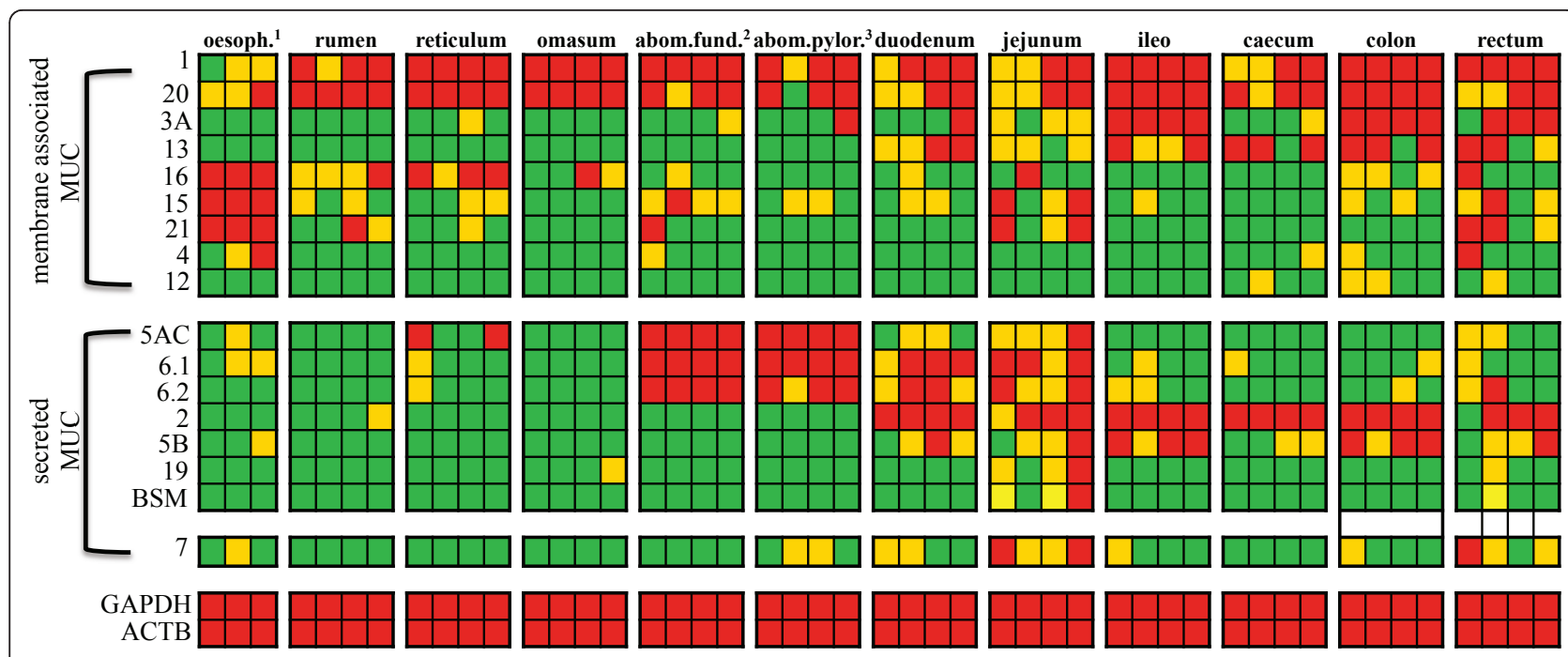

Figure 3 Transcription profile of bovine mucins in the gastrointestinal tract. Transcription profile of membrane associated and secreted mucins in the gastro-intestinal tract. PCR on CDNA samples was used to characterize the transcription profile of membrane associated and secreted mucins in twelve regions of the gastro-intestinal tract. Squares represent PCR products of individual animals $(n=3$ in oesophagus; $n=$ 4 in all other tissues). Presence and intensity of gel bands were analyzed using ImageQuant ${ }^{\mathrm{T}} 350$ and are reported as absent (green), present weak (yellow), and present strong (red). Glyceraldehyde 3-phosphate (GAPDH) and $\beta$-actin (ACTB) were used to control the quality of the synthesized cDNA in all the samples.

MUC19 was also found to be weakly transcribed in the omasum of one animal, whereas the BSM transcript was not detectable in this sample. The secreted non gelforming mucin MUC7 was transcribed in a limited number of tissues analyzed. Finally, for the two control genes, GAPDH and $A C T B$, strong bands were obtained in all the samples analyzed.

\section{Discussion}

The current study identified 15 bovine mucin-encoding genes in the cattle genome (assembly Btau_4.0), including nine membrane-associated mucins and six secreted ones. Compared to human, no homologues could be identified for $M U C 3 B, M U C 8$ and $M U C 17$. This feature is not unique to cattle since previous studies showed that these three mucins were also absent in rodents $[25,27]$. In humans, the two genes encoding MUC3A and $M U C 3 B$ show a high identity at nucleotide level both in the intronic and exonic sequences, suggesting that they are the product of a rather recent gene duplication after the divergence of humans and rodents [25]. This hypothesis would explain why MUC3A and $M U C 3 B$ are found in chimpanzee, which diverged from humans later compared to rodents, but not in cows and other ruminants which diverged from humans earlier than rodents [45]. In addition, high similarity among human MUC17 and rodent MUC3 has previously been shown [27]. Although it has previously been suggested that human MUC17 is the true structural homologue of rodent MUC3 [27], the conserved localization of the
MUC3 gene upstream of MUC12 and MUC17 in humans and upstream of MUC12 in mice and cattle, suggests that MUC3 in the latter two species is the homologue of human MUC3 rather than of MUC17. In regard to $M U C 8$, BLAST analysis suggested that humans are the only species having this gene in their genome (data not shown).

As previously stated, sequencing mucin genes is technically difficult due to their size and the large number of repetitive sequences in the PTS regions. Therefore, some of the mucin gene and mRNA sequences are still incomplete. For example for MUC6, sequence information for the central PTS region is still missing. Similar problems were encountered for $M U C 3 A, M U C 7$, MUC12 and MUC13.

In humans, two clusters of mucins have been previously described. MUC3, MUC12 and MUC17, encoding membrane bound mucins, are clustered on human chromosome 7q22 [27], while MUC6, MUC2, MUC5AC, and $M U C 5 B$, encoding secreted gel-forming mucins, are clustered on human chromosome 11p15 [46]. With the exception of MUC17, for which no bovine homologue has been identified, both gene clusters appear to be maintained in cattle, on bovine chromosome BTA25 and BTA29, respectively. This conservation has also been described for the MUC6, MUC2, MUC5AC and MUC5B gene cluster in mice [36].

Alignment of the bovine and human sequences showed that, although the characteristic regions and domains of mucin glycoproteins are generally conserved 
between these species, the overall sequence similarity is very low with values included between $16.9 \%$ and $68.2 \%$. The reason for this low similarity is probably related to the presence of the PTS regions, which are characteristic of these proteins [47]. This is consistent with previous studies that compared human and rodent mucins $[26,48,49]$. In general, domain architecture of the membrane-associated mucins was found to be similar between humans and cattle, while the protein architecture of the gel-forming mucins appeared to be less conserved. The bovine MUC5AC, MUC5B, and MUC6 proteins did not show the VWD-C8-TIL-VWC domain organization which is typical for the $\mathrm{N}$-terminus of all human secreted gel-forming mucins. Bovine MUC5AC contained no TIL domains over its entire length, while bMUC6 was shown to have no VWC domains. However, all the domains responsible for the oligomerization of these molecules, such as VWD and cystein rich domains, are present. In view of the sequence variation detected compared to the human MUC genes, it is still unclear what the variation is in the MUC genes of different cattle breeds. High variability in the number of VNTRs and the extent of VNTR polymorphism within and across cattle breeds has only been reported for the MUC1 gene [50,51]. The level of allelic variance for the other bovine $M U C$ genes is still unclear.

$B S M$ is one of the few mucins previously isolated and studied in cattle $[33,34,43]$. It is located on chromosome 5 and high sequence similarities have been shown with the C-terminal part of porcine and ovine submaxillary mucins (PSM and OSM). In 2004 [44,52], human and mouse MUC19 were reported as new members of the gel-forming mucin family. Their sequence similarity, similar expression pattern (majorly in salivary glands), and chromosomal location compared to BSM and PSM suggested that they were potential homologues. The previously reported BSM sequence lacked the N-terminal conserved protein domains present in PSM and other MUC19 sequences [34], suggesting that it was not complete. In the current study, a new mRNA and corresponding gDNA sequence (bMUC19) was identified showing homology to the 5' part of hMUC19. This gDNA sequence is located approximately $9 \mathrm{~kb}$ upstream of the BSM gene on chromosome 5. The genomic localization and the almost identical transcription profile of both genes would suggest that BSM and this newly identified bMUC19 are actually part of one gene encoding the bovine MUC19 homologue.

At the moment, mechanisms regulating human mucin transcription are largely unknown and promotor sequences have not been identified for all human $M U C$ genes. The transcription factors NF-kappaB and AP-1 seem to be involved in the regulation of a number of human $M U C$ genes, including the membrane bound mucin $M U C 1$ [53] and the secreted mucins $M U C 2$ [54-56], MUC5AC [57-59], MUC5B [60,61] and MUC6 [62]. In cattle, promotor sequences are available only for MUC1, MUC3A, MUC5B, MUC12, MUC15, MUC20 and $M U C 21$. In silico promotor analysis showed potential binding sites for AP-1 in the secreted mucin $M U C 5 B$ and for AP-1 and c-Rel/NF-kappaB in all the membrane bound mucins, with the exception of $M U C 1$ (data not shown). As more sequence data become available, it will be interesting to investigate if different regulatory mechanisms exist within the different classes of mucin genes and whether they can affect (patho) physiological conditions.

Transcriptional analysis of the 15 identified mucin genes showed that most of them are transcribed in the GI tract of cattle. Although some individual differences were observed, in general, for the membrane associated mucins, MUC1 and MUC2O transcripts were detected in all the tissues examined, whereas MUC3 seemed to be mainly produced in the intestinal tissues. This is consistent with the observations made in humans $[35,63]$. MUC15, MUC16 and MUC21, on the other hand, were the major membrane-associated mucins found in the oesophagus. A search of the human EST dataset revealed the presence of $M U C 15$ and MUC21 encoding ESTs derived from an oesophageal cDNA library (data not shown), suggesting that their transcription pattern might be conserved. Concerning the secreted mucins, $M U C 5 A C$ and MUC6 were primarily transcribed in the abomasum, whereas $M U C 2$ and $M U C 5 B$ were the main secreted mucins in the intestinal tissues. This is largely in agreement with the situation in humans, except for $M U C 5 B$, for which evidence in the human intestine has only been reported in colonic tissue $[64,65]$. In contrast to our results in cattle, the human $M U C 5 B$ is also found in the oesophagus, where it is transcribed in the oesophageal submucosal glands (SMGs). SMGs are known to differ in distribution and structures among species [66], so it is possible that the oesophageal samples collected in this study did not include regions rich in these glands. Alternatively, it is also possible that in cattle SMGs do not produce MUC5B. Interestingly, secreted mucins were not found to be produced in the pre-stomachs. Since secreted gel-forming mucins are widely acknowledged as an important component in the formation of viscoelastic mucus in stomach and intestine to protect the mucosal epithelium against acidic and proteolytic damages [67], the absence of gastric juices, hydrochloric acid and digestive enzymes in the pre-stomach may explain this lack of expression. On the other hand, the pattern of gene expression of both the membrane bound and secreted mucins in the prestomachs was very similar to the pattern observed in the oesophagus, which could be due to the common origin 
that these organs have during bovine embryogenesis [68]. Although the transcription pattern of bovine mucins in the GI tract is genererally similar to what is observed in humans, transcription levels do not always correspond with protein levels and different factors can influence the functional properties of the mature protein. Alternative splicing can generate different isoforms with different tissue distributions and properties. For several human membrane associated mucins, multiple isoforms have been identified, some of which are secreted $[22,23,69]$. In addition, posttranslational proteolysis of the SEA domain can also release the extracellular domain of membrane bound mucins into the mucus layer $[20,29]$. Since the large PTS regions constitute $\mathrm{O}$-glycosylation sites, variance in the number, length and sequence of the repeats can impact the extent and type of glycosylation and thus the biological functions of mucins that are largely defined by their carbohydrate constitution [4]. Only a limited amount of studies have been performed to investigate these aspects in bovine mucins $[50,70]$ and future studies will need to include these different factors influencing mucin biology.

\section{Conclusions}

This study provides the first characterization of the mucin gene family in cattle and their transcriptional distribution in the GI tract. Homologues were identified for all members of the human mucin family, with the exception of human $M U C 3 B, M U C 8$ and MUC17. The protein domain architecture of the membrane-associated mucins was found to be conserved between humans and cattle, while protein architecture of the gel-forming mucins appeared to be less conserved. Analysis of the transcription profile showed that the secreted mucins were transcribed from the abomasum onwards, whereas the membrane associated mucins MUC1 and MUC20 were transcribed throughout the whole GI tract.

\section{Acknowledgements \\ This research was funded by a PhD grant of the 'Institute for the Promotion of Innovation through Science and Technology in Flanders' (IWT-Vlaanderen) (IWT-SB/61028/Hoorens) and supported by the 'Fund for Scientific Research Flanders' (F.W.O. 1.5.005.07) and the Concerted Research Actions of Ghent University (GOA) \\ Author details \\ 'Department of Virology, Parasitology and Immunology, Faculty of Veterinary Medicine, Ghent University, Salisburylaan 133, 9820 Merelbeke, Belgium. ${ }^{2}$ Bovine Functional Genomics Laboratory, Animal and Natural Resources Institute, USDA-ARS, 10300 Baltimore Avenue, Beltsville, MD 20705, USA. ${ }^{3}$ Department Biosystems, Division Gene Technology, Faculty of Bioscience Engineering, K.U. Leuven, Kasteelpark Arenberg 30, 3001 Leuven, Belgium.}

\section{Authors' contributions}

This article is a part of PH's PhD thesis. MR helped in performing the transcription studies. RL contributed to the genome mining. BG, EC, JV and PG conceived and designed the project. All authors read and approved the final manuscript.
Received: 20 October 2010 Accepted: 7 March 2011

Published: 7 March 2011

\section{References}

1. Desseyn $J \mathrm{~L}$, Tetaert $\mathrm{D}$, Gouyer $\mathrm{V}$ : Architecture of the large membranebound mucins. Gene 2008, 410(2):215-222

2. Hattrup $\mathrm{CL}$, Gendler SJ: Structure and function of the cell surface (tethered) mucins. Annu Rev Physiol 2008, 70:431-457.

3. Linden SK, Sutton P, Karlsson NG, Korolik V, McGuckin MA: Mucins in the mucosal barrier to infection. Mucosal Immunol 2008, 1(3):183-197.

4. Thornton DJ, Rousseau K, McGuckin MA: Structure and function of the polymeric mucins in airways mucus. Annu Rev Physiol 2008, 70:459-486.

5. Zalewska A, Zwierz K, Zolkowski K, Gindzienski A: Structure and biosynthesis of human salivary mucins. Acta Biochim Pol 2000, 47(4):1067-1079.

6. Shankar V, Pichan P, Eddy RL, Tonk V, Nowak N, Sait SNJ, Shows TB, Schultz RE, Gotway G, Elkins RC, et al: Chromosomal localization of a human mucin gene (MUC8) and cloning of the CDNA corresponding to the carboxy terminus. American Journal of Respiratory Cell and Molecular Biology 1997, 16(3):232-241

7. Shankar V, Gilmore MS, Elkins RC, Sachdev GP: A Novel Human Airway Mucin Cdna Encodes a Protein with Unique Tandem-Repeat Organization. Biochemical Journal 1994, 300:295-298.

8. Bafna S, Kaur S, Batra SK: Membrane-bound mucins: the mechanistic basis for alterations in the growth and survival of cancer cells. Oncogene 2010, 29(20):2893-2904.

9. Moniaux N, Escande F, Porchet N, Aubert JP, Batra SK: Structural organization and classification of the human mucin genes. Front Biosci 2001, 6:D1192-1206.

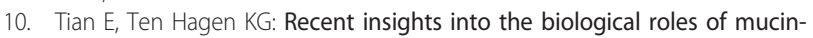
type O-glycosylation. Glycoconj J 2009, 26(3):325-334.

11. Lang T, Hansson GC, Samuelsson T: Gel-forming mucins appeared early in metazoan evolution. Proc Natl Acad Sci USA 2007, 104(41):16209-16214.

12. Perez-Vilar J, Eckhardt AE, DeLuca A, Hill RL: Porcine submaxillary mucin forms disulfide-linked multimers through its amino-terminal D-domains. J Biol Chem 1998, 273(23):14442-14449.

13. Axelsson MA, Asker N, Hansson GC: O-glycosylated MUC2 monomer and dimer from LS 174T cells are water-soluble, whereas larger MUC2 species formed early during biosynthesis are insoluble and contain nonreducible intermolecular bonds. J Biol Chem 1998, 273(30):18864-18870

14. Perez-Vilar J, Hill RL: The structure and assembly of secreted mucins. J Biol Chem 1999, 274(45):31751-31754.

15. Godl K, Johansson ME, Lidell ME, Morgelin M, Karlsson H, Olson FJ, Gum JR $\mathrm{Jr}$, Kim YS, Hansson GC: The N terminus of the MUC2 mucin forms trimers that are held together within a trypsin-resistant core fragment. J Biol Chem 2002, 277(49):47248-47256.

16. Sheehan JK, Howard M, Richardson PS, Longwill T, Thornton DJ: Physical characterization of a low-charge glycoform of the MUC5B mucin comprising the gel-phase of an asthmatic respiratory mucous plug. Biochem J 1999, 338(Pt 2):507-513.

17. Sheehan JK, Brazeau C, Kutay S, Pigeon H, Kirkham S, Howard M, Thornton DJ: Physical characterization of the MUC5AC mucin: a highly oligomeric glycoprotein whether isolated from cell culture or in vivo from respiratory mucous secretions. Biochem J 2000, 347(Pt 1):37-44.

18. Levine MJ, Reddy MS, Tabak LA, Loomis RE, Bergey EJ, Jones PC, Cohen RE, Stinson MW, Al-Hashimi I: Structural aspects of salivary glycoproteins. J Dent Res 1987, 66(2):436-441.

19. Dekker J, Rossen JW, Buller HA, Einerhand AW: The MUC family: an obituary. Trends Biochem Sci 2002, 27(3):126-131.

20. Wreschner DH, McGuckin MA, Williams SJ, Baruch A, Yoeli M, Ziv R, Okun L, Zaretsky J, Smorodinsky N, Keydar I, et al: Generation of ligand-receptor alliances by "SEA" module-mediated cleavage of membrane-associated mucin proteins. Protein Sci 2002, 11(3):698-706.

21. Macao B, Johansson DG, Hansson GC, Hard T: Autoproteolysis coupled to protein folding in the SEA domain of the membrane-bound MUC1 mucin. Nat Struct Mol Biol 2006, 13(1):71-76.

22. Choudhury A, Moniaux N, Ringel J, King J, Moore E, Aubert JP, Batra SK: Alternate splicing at the 3 '-end of the human pancreatic tumorassociated mucin MUC4 cDNA. Teratog Carcinog Mutagen 2001, 21(1):83-96. 
23. Pallesen LT, Berglund L, Rasmussen LK, Petersen TE, Rasmussen JT: Isolation and characterization of MUC15, a novel cell membrane-associated mucin. Eur J Biochem 2002, 269(11):2755-2763.

24. Ensembl. [http://www.ensembl.org/index.html].

25. Pratt WS, Crawley S, Hicks J, Ho J, Nash M, Kim YS, Gum JR, Swallow DM: Multiple transcripts of MUC3: evidence for two genes, MUC3A and MUC3B. Biochem Biophys Res Commun 2000, 275(3):916-923.

26. Shekels LL, Hunninghake DA, Tisdale AS, Gipson IK, Kieliszewski M, Kozak CA, Ho SB: Cloning and characterization of mouse intestinal MUC3 mucin: $3^{\prime}$ sequence contains epidermal-growth-factor-like domains. Biochem J 1998, 330(Pt 3):1301-1308.

27. Gum JR Jr, Crawley SC, Hicks JW, Szymkowski DE, Kim YS: MUC17, a nove membrane-tethered mucin. Biochem Biophys Res Commun 2002, 291(3):466-475.

28. Shankar V, Pichan P, Eddy RL Jr, Tonk V, Nowak N, Sait SN, Shows TB, Schultz RE, Gotway G, Elkins RC, et al: Chromosomal localization of a human mucin gene (MUC8) and cloning of the cDNA corresponding to the carboxy terminus. Am J Respir Cell Mol Biol 1997, 16(3):232-241.

29. Pallesen $L T$, Andersen $M H$, Nielsen $R L$, Berglund $L$, Petersen $T E$, Rasmussen LK, Rasmussen JT: Purification of MUC1 from bovine milk-fat globules and characterization of a corresponding full-length CDNA clone. J Dairy Sci 2001, 84(12):2591-2598.

30. Perucatti A, Floriot S, Di Meo GP, Soglia D, Rullo R, Maione S, Incarnato D, Eggen A, Sacchi P, Rasero R, et al: Comparative FISH mapping of mucin 1, transmembrane (MUC1) among cattle, river buffalo, sheep and goat chromosomes: comparison between bovine chromosome 3 and human chromosome 1. Cytogenet Genome Res 2006, 112(1-2):103-105.

31. Rasero R, Bianchi L, Cauvin E, Maione S, Sartore S, Soglia D, Sacchi P: Analysis of the sheep MUC1 gene: structure of the repetitive region and polymorphism. J Dairy Sci 2007, 90(2):1024-1028.

32. Pallesen $L T$, Pedersen $L R$, Petersen $T E$, Knudsen $C R$, Rasmussen JT: Characterization of human mucin (MUC15) and identification of ovine and caprine orthologs. J Dairy Sci 2008, 91(12):4477-4483.

33. Bhargava AK, Woitach JT, Davidson EA, Bhavanandan VP: Cloning and cDNA sequence of a bovine submaxillary gland mucin-like protein containing two distinct domains. Proc Natl Acad Sci USA 1990, 87(17):6798-6802.

34. Jiang W, Gupta D, Gallagher D, Davis S, Bhavanandan VP: The central domain of bovine submaxillary mucin consists of over 50 tandem repeats of 329 amino acids. Chromosomal localization of the BSM1 gene and relations to ovine and porcine counterparts. Eur J Biochem 2000, 267(8):2208-2217.

35. Corfield AP, Myerscough N, Longman R, Sylvester P, Arul S, Pignatelli M: Mucins and mucosal protection in the gastrointestinal tract: new prospects for mucins in the pathology of gastrointestinal disease. Gut 2000, 47(4):589-594.

36. Escande F, Porchet N, Bernigaud A, Petitprez D, Aubert JP, Buisine MP: The mouse secreted gel-forming mucin gene cluster. Biochim Biophys Acta 2004, 1676(3):240-250.

37. National Center for Biotechnology Information. [http://www.ncbi.nlm.nih gov/].

38. Center for Medical Genetics. [http://medgen.ugent.be/CMGG].

39. Simple Modular Architecture Research Tool. [http://smart.embl-heidelberg. de]

40. SignalP 3.0 server. [http://www.cbs.dtu.dk/services/SignalP/].

41. Primer3 Input (version 0.4.0). [http://frodo.wi.mit.edu]

42. GENSCAN. [http://genes.mit.edu/GENSCAN.html]

43. Jiang W, Woitach JT, Keil RL, Bhavanandan VP: Bovine submaxillary mucin contains multiple domains and tandemly repeated non-identical sequences. Biochem J 1998, 331(Pt 1):193-199.

44. Chen Y, Zhao YH, Kalaslavadi TB, Hamati E, Nehrke K, Le AD, Ann DK, Wu R: Genome-wide search and identification of a novel gel-forming mucin MUC19/Muc19 in glandular tissues. Am J Respir Cell Mol Biol 2004, 30(2):155-165.

45. McKnight DA, Fisher LW: Molecular evolution of dentin phosphoprotein among toothed and toothless animals. BMC Evol Biol 2009, 9:299.

46. Vincent $A$, Perrais $M$, Desseyn $J L$, Aubert JP, Pigny P, Van Seuningen I: Epigenetic regulation (DNA methylation, histone modifications) of the 11 15 mucin genes (MUC2, MUC5AC, MUC5B, MUC6) in epithelial cancer cells. Oncogene 2007, 26(45):6566-6576.
47. Desseyn $\lrcorner L$, Aubert JP, Porchet N, Laine A: Evolution of the large secreted gel-forming mucins. Mol Biol Evol 2000, 17(8):1175-1184

48. Vos HL, de Vries Y, Hilkens J: The mouse episialin (Muc1) gene and its promoter: rapid evolution of the repetitive domain in the protein. Biochem Biophys Res Commun 1991, 181(1):121-130.

49. Gum JR Jr, Hicks JW, Lagace RE, Byrd JC, Toribara NW, Siddiki B, Fearney FJ, Lamport DT, Kim YS: Molecular cloning of rat intestinal mucin. Lack of conservation between mammalian species. J Biol Chem 1991, 266(33):22733-22738

50. Sando L, Pearson R, Gray C, Parker P, Hawken R, Thomson PC, Meadows JR, Kongsuwan K, Smith S, Tellam RL: Bovine Muc1 is a highly polymorphic gene encoding an extensively glycosylated mucin that binds bacteria. $J$ Dairy Sci 2009, 92(10):5276-5291.

51. de Souza FR, Dentillo DB, Meola J, Biase FH, Andrea MV, Vozzi PA, Lobo RB, Martelli LR: The polymorphism in MUC1 gene in Nelore cattle. J Anim Breed Genet 2007, 124(1):42-46.

52. Culp DJ, Latchney LR, Fallon MA, Denny PA, Denny PC, Couwenhoven RI, Chuang S: The gene encoding mouse Muc19: CDNA, genomic organization and relationship to Smgc. Physiol Genomics 2004, 19(3):303-318.

53. Thompson EJ, Shanmugam K, Hattrup CL, Kotlarczyk KL, Gutierrez A, Bradley JM, Mukherjee P, Gendler SJ: Tyrosines in the MUC1 cytoplasmic tail modulate transcription via the extracellular signal-regulated kinase $1 / 2$ and nuclear factor-kappaB pathways. Mol Cancer Res 2006, 4(7):489-497.

54. Song S, Byrd JC, Mazurek N, Liu K, Koo JS, Bresalier RS: Galectin-3 modulates MUC2 mucin expression in human colon cancer cells at the level of transcription via AP-1 activation. Gastroenterology 2005, 129(5):1581-1591.

55. Song S, Byrd JC, Koo JS, Bresalier RS: Bile acids induce MUC2 overexpression in human colon carcinoma cells. Cancer 2005, 103(8):1606-1614

56. Lee HY, Crawley S, Hokari R, Kwon S, Kim YS: Bile acid regulates MUC2 transcription in colon cancer cells via positive EGFR/PKC/Ras/ERK/CREB, $\mathrm{PI3K} / \mathrm{Akt} / \mathrm{lkappaB} / \mathrm{NF}-\mathrm{kappaB}$ and p38/MSK1/CREB pathways and negative JNK/C-Jun/AP-1 pathway. Int J Oncol 36(4):941-953.

57. Voynow JA, Rubin BK: Mucins, mucus, and sputum. Chest 2009, 135(2):505-512.

58. Fujisawa T, Velichko $S$, Thai $P$, Hung LY, Huang F, Wu R: Regulation of airway MUC5AC expression by IL-1 beta and IL-17A; the NF-kappaB paradigm. J Immunol 2009, 183(10):6236-6243

59. Li S, Zhou X: Construction of luciferase reporter gene vector for human MUC5AC gene promoter and analysis of its transcriptional activity. Zhong Nan Da Xue Xue Bao Yi Xue Ban 35(8):792-799.

60. Fujisawa $T$, Chang MM, Velichko $S$, Thai $P$, Hung LY, Huang F, Phuong $N$, Chen Y, Wu R: NF-\{kappa\} B Mediates IL-1ss- and IL-17A-induced MUC5B Expression in the Airway Epithelial Cells. Am J Respir Cell Mol Biol

61. Van Seuningen I, Perrais M, Pigny P, Porchet N, Aubert JP: Sequence of the 5 -flanking region and promoter activity of the human mucin gene MUC5B in different phenotypes of colon cancer cells. Biochem J 2000, 348(Pt 3):675-686.

62. Sakai $H$, Jinawath A, Yamaoka S, Yuasa Y: Upregulation of MUC6 mucin gene expression by NFkappaB and Sp factors. Biochem Biophys Res Commun 2005, 333(4):1254-1260.

63. Vanklinken BJW, Dekker J, Buller HA, Einerhand AWC: Mucin Gene Structure and Expression - Protection Vs Adhesion. American Journal of Physiology-Gastrointestinal and Liver Physiology 1995, 32(5):G613-G627.

64. Reid CJ, Harris A: Developmental expression of mucin genes in the human gastrointestinal system. Gut 1998, 42(2):220-226.

65. van Klinken BJ, Dekker J, van Gool SA, van Marle J, Buller HA, Einerhand AW: MUC5B is the prominent mucin in human gallbladder and is also expressed in a subset of colonic goblet cells. Am J Physiol 1998, 274(5 Pt 1):G871-878.

66. Long JD, Orlando RC: Esophageal submucosal glands: structure and function. Am J Gastroenterol 1999, 94(10):2818-2824.

67. Phillipson M, Johansson MEV, Henriksnas J, Petersson J, Gendler SJ, Sandler S, Persson AEG, Hansson GC, Holm L: The gastric mucus layers: constituents and regulation of accumulation. American Journal of Physiology-Gastrointestinal and Liver Physiology 2008, 295(4):G806-G812.

68. Latshaw WK: Veterinary developmental anatomy: a clinically oriented approach. B.C. Decker; 1987. 
69. Baruch A, Hartmann M, Yoeli M, Adereth Y, Greenstein S, Stadler Y, Skornik Y, Zaretsky J, Smorodinsky NI, Keydar I, et al: The breast cancerassociated MUC1 gene generates both a receptor and its cognate binding protein. Cancer Res 1999, 59(7):1552-1561.

70. Pallesen $L T$, Pedersen $L R$, Petersen TE, Rasmussen JT: Characterization of carbohydrate structures of bovine MUC15 and distribution of the mucin in bovine milk. J Dairy Sci 2007, 90(7):3143-3152.

71. Mucin Biology Group Mucin Database. [http://www.medkem.gu.se/ mucinbiology].

doi:10.1186/1471-2164-12-140

Cite this article as: Hoorens et al:: Genome wide analysis of the bovine mucin genes and their gastrointestinal transcription profile. $B M C$

Genomics 2011 12:140.

Submit your next manuscript to BioMed Central and take full advantage of:

- Convenient online submission

- Thorough peer review

- No space constraints or color figure charges

- Immediate publication on acceptance

- Inclusion in PubMed, CAS, Scopus and Google Scholar

- Research which is freely available for redistribution

Submit your manuscript at www.biomedcentral.com/submit 